\title{
Structural Quality of GaSb/GaAs Quantum Dots for Solar Cells Analyzed by Electron Microscopy Techniques
}

\author{
N. Fernández-Delgado ${ }^{1}$, M. Herrera ${ }^{1}$, N. Baladés ${ }^{1}$, J. S. James ${ }^{2}$, A. Krier ${ }^{2}$, H. Fujita $^{3}$ and S. I. Molina ${ }^{1}$ \\ ${ }^{1}$ Department of Material Science, Metallurgical Engineering and Inorganic Chemistry, University of Cádiz, \\ 11510, Puerto Real, Cádiz, Spain. \\ 2.Physics Department, Lancaster University, Lancaster, LA1 4YB, UK. \\ ${ }^{3 .}$ Magnetic Sensors Process Technology \& Development Department, Asahi-Kasei Microdevices, Japan.
}

Nowadays, the research on environmentally friendly and renewable energy sources is of primary importance because of the continuous reduction in the reserve of fossil fuel and the increase in the concerns of global warming. Taking advantage of solar energy for this purpose it is acquiring increasing importance in the last decades. Nanostructured semiconductor materials such as quantum dots (QDs) and quantum rings (QRs) attract considerable interest in this field because of their potential to considerably improve the performance of III-V single- and multi-junction solar cells [1,2]. In particular, type II GaSb/GaAs QDs can largely extend the spectral response beyond the visible out towards $1.4 \mu \mathrm{m}$ providing a near optimum band gap for concentrator solar cell applications [3]. GaSb is a narrow direct band gap semiconductor often used in optoelectronics to fabricate lasers, photodetectors, etc. and it is considered a good candidate for thermophotovoltaic (TPV) applications [4,5].

However, the main problem in the design of $\mathrm{GaSb} / \mathrm{GaAs}$ devices is the large lattice mismatch of $7.8 \%$ between the $\mathrm{GaSb}$ active layers and the GaAs substrate, which usually leads to plastic relaxation mechanisms such as the formation of misfit or threading dislocations [6]. These defects may cause additional electronic states within the band gap leading to nonradiative recombination phenomena that would affect the electronic and optical properties [7,8]. In order to minimize these non-desirable effects, different strategies have been proposed, such as optimizing the growth conditions in order to obtain an interfacial misfit dislocation array (IMF) [6,9]. Diffusion and/or segregation effects are also frequent phenomena in the growth of semiconductor nanostructured materials that may prevent obtaining the designed optoelectronic properties [10]. Because of this, the analysis at atomic scale of the structural properties and the composition distribution of these materials is essential in order to understand and optimize the performance of future devices.

In this communication, we have studied by transmission electron microscopy (TEM) the structural properties of a heterostructure of layers of GaSb QDs for solar cell applications. The sample has been grown by molecular beam epitaxy (MBE) on a GaAs substrate. Initially, we have analysed the sample by diffraction contrast $220 \mathrm{BF}$, as shown in Fig. 1. In this micrograph, strain contrasts due to the 3D geometry of the QDs are observed. It should be highlighted that extended structural defects such as threading dislocation have not been found, which is important for optimized functional properties. We have also analysed the sample by diffraction contrast $002 \mathrm{DF}$, which is sensitive to the material composition. As it can be observed in Fig. 2, the GaSb layers appear with bright contrasts. As shown in the inset, we have found some contrast (marked in the image) that could be related to the 3D structures. However, at this magnification they can not be clearly resolved. Aberration corrected atomic scale high angle annular dark field scanning (HAADF-S) TEM analysis are in progress in order to study in detail the size and shape of the grown nanostructures, the composition distribution and the existence of misfit dislocations. 


\section{References:}

[1] A. Nozik, Physica E 14 (2002) p. 115.

[2] P. J. Carrington, M. C. Wagener, J. R. Botha, A. M. Sanchez, A. Krier, Appl. Phys. Lett. 101 (2012) 231101.

[3] P. J. Carrington, A. S. Mahajumi , M. C. Wagener, J. R. Botha, Q. Zhuang, A. Krier, Physica 407 (2012) p. 1493.

[4] S. K. Chou, W. M. Yang, K. J. Chua, J. Li, K. L. Zhang, Applied Energy 91 (2012) p. 304.

[5] L. Tang, H. Ye, J. Xu, Solar En. Mat. \& Solar Cells, 122 (2014) p. 94.

[6] B. Laghumavarapu, A. Moscho, A. Khoshakhlagh, M. El-Emawy, L. F. Lester, D. L. Huffaker, Appl. Phys. Lett. 90 (2007), 173125.

[7] R. D. Schaller, V.I. Klimov, Phys. Rev. Lett. 92 (2004) 186601.

[8] S. Lopatin, S. J. Pennycook, J. Narayan, G. Duscher, Appl. Phys. Lett. 81 (2002) p. 15.

[9] A. M. Rocher, Solid State Phenom. 19/20 (1991) p. 363.

[10] L. Mancini, Y. Fotana, S. Conesa-Boj, I. Blum, F. Vurpillot, L. Francavigilia, E. Russo-Averchi, M. Heiss, J. Arbiol, M. I. Fontcuberta, L. Rigutti, Appl. Phys. Lett. 105 (2014) 4904952.

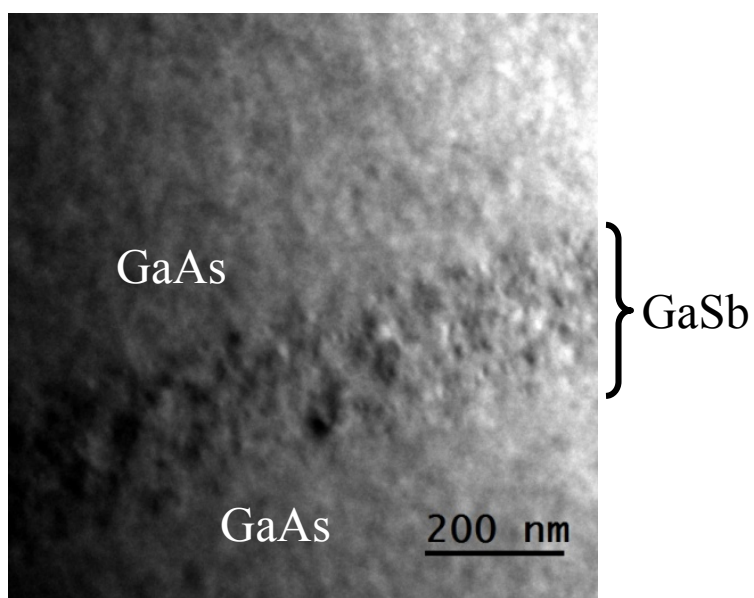

Figure 1. TEM image of the sample analyzed by diffraction contrast $220 \mathrm{BF}$.

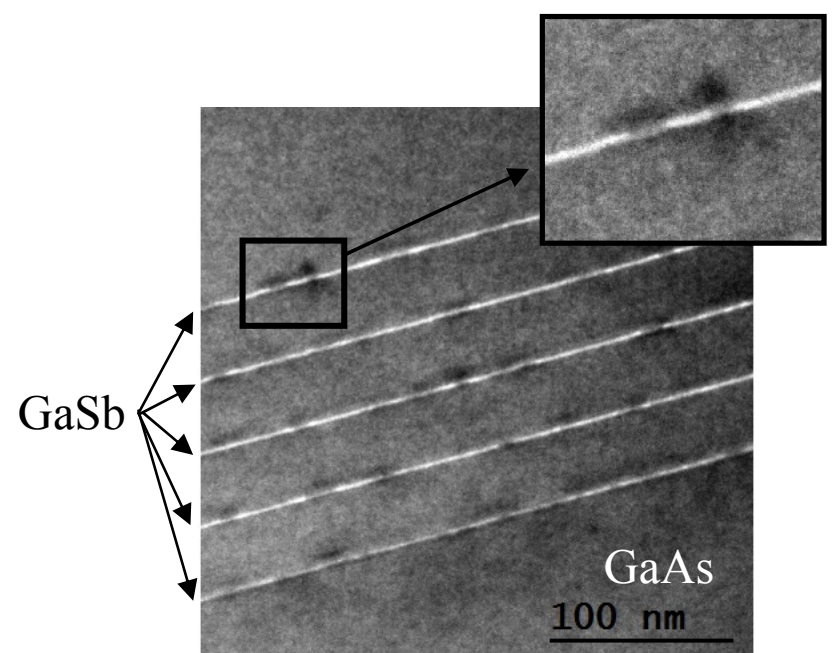

Figure 2. TEM image of the sample analyzed by diffraction contrast 002 DF. 